\title{
Transcatheter embolization of bronchial arteries in the treatment of haemoptysis
}

\author{
Vinko Vidjak ${ }^{1}$, Karlo Novačić ${ }^{1}$, Andrija Hebrang${ }^{1}$, Ivica Mažuranić ${ }^{2}$ \\ Miroslav Samaržija ${ }^{3}$, Spomenka Ljubić ${ }^{4}$, Tomislav Breitenfeld ${ }^{5}$, Branimir Klasić ${ }^{6}$
}

\begin{abstract}
${ }^{1}$ Department of Diagnostic and Interventional Radiology, Merkur University Hospital,
${ }^{2}$ Department of Thoracic Radiology, Jordanovac University Hospital for Lung Diseases, ${ }^{3}$ Postintensive Care Department, Jordanovac University Hospital for Lung Diseases, ${ }^{4}$ Metabolic Department, Vuk Vrhovac University Clinic, ${ }^{5}$ University Department of Neurology, Sestre Milosrdnice Universtity Hospital, ' ${ }^{6}$ Department of Radiology, Hospital for Lung Diseases,

${ }^{1-5}$ School of Medicine, University of Zagreb, Zagreb, Croatia
\end{abstract}

Background. Massive haemoptysis is a clinical state characterized by the expectoration of blood at a rate of 300-600 mL/24 h, thus causing life-threatening asphyxia. The aim of our study is to review use of transcatheter bronchial artery embolization (BAE) in the treatment of massive haemoptysis.

Materials and methods. Series of 11 patients with the clinical picture of massive haemoptysis was referred to our hospital for digital subtraction angiography and BAE within a 33 months period. There were 8 male (aged 43-69, mean age 56) and 3 female patients (aged 63-65, mean age 64). Aortography of thoracic aorta was initially performed in all patients, followed by selective angiography of bronchial and intercostal arteries, and intercostobronchial tree as indicated. A selective arterial embolization was done in 9 patients (9 primary and 3 secondary embolizations). The embolization was performed under fluoroscopy control by manual injection of the mixture of contrast solution $(1 \mathrm{ccm})$ and embolization material, Embosphere (BioSphere Medical Inc., MA, USA), particle size 350-500 $\mu \mathrm{m}$.

Results. Bronchiectasis was the most common cause of bleeding (45.4\%), while hypervascularization and intensive parenchymal opacification were the most frequent angiographic indicators of bleeding (100\%), followed by tortuous and hypertrophic arteries (72.7\%). Primary BAE proved successful in $81.9 \%$ and secondary BAE performed within 24 months in $33.3 \%$ of patients, whereas the tertiary (operative) treatment was required in $22.2 \%$ of patients. In $44.4 \%$ of patients, BAE was associated with only mild discomforts, like pain and cough.

Conclusions. BAE is a reliable and minimally invasive method in the management of massive haemoptysis. Therefore, it should be considered as the primary method of the treatment or as a procedure for the stabilization the patient before the surgery.

Key words: massive haemoptysis; bronchial artery; embolization 


\section{Introduction}

Massive haemoptysis is the expectoration of blood at a rate of 300-600 mL/24 h. If left untreated, it can lead to asphyxia and death. Massive haemoptysis accounts for $5 \%$ of all haemoptysis cases and is associated with a high mortality rate (up to $75 \%$ ). If massive haemoptysis is treated conservatively, mortality rate ranges from $50 \%$ to $85 \% .^{1-4}$ Tuberculosis and sarcoidosis are the most common causes of massive haemoptysis. In $90 \%$ of cases, haemoptysis originates from bronchial arteries, whereas only a minor portion arises from non-bronchial (pulmonary and systemic) circulation. ${ }^{2}$ The bronchial artery embolization (BAE) is a percutaneous transcatheter minimally invasive procedure, which has proved successful in the management of massive haemoptysis and is associated with a lower mortality rate as compared with the surgical treatment $(7.1 \%-18.2 \%) .2,5,6$ The aim is to present our results in the management of haemoptysis by the use of transcatheter BAE.

\section{Patients and methods}

During 33 months, 11 patients were referred to our hospital for digital subtraction angiography (DSA) and BAE because of the clinical picture of massive haemoptysis. Bronchiectasis was the cause of haemorrhage in $5(45.4 \%)$, lung tuberculosis in 3 $(27.3 \%)$, aspergilloma in $2(18.2 \%)$ patients, and carcinoma in $1(9.1 \%)$ patient.

DSA was recommended by interdisciplinary consultation (pulmonologist, thoracic surgeon and interventional radiologist). DSA and BAE were preceded by lung X-ray, computed tomography of the thorax and bronchoscopy to identify the localization of bleeding and type of lesions.

Bronchoscopy proved inadequate in 5 patients because of the procedure intoler- ance due to the patients' poor general condition and poor visualization of the site of bleeding. An informed consent was obtained from all patients prior to the procedure. There were 8 male (aged 43-69, mean age 56) and 3 female (aged 63-65, mean age 64) patients.

Prior to DSA, patients were administered diazepams, 5 mg i.v. (Apaurin, Krka, Novo Mesto, Slovenia) for mild sedation, along with monitoring of vital functions. DSA was performed by Seldinger technique via common femoral artery, by using diagnostic pigtail and selective 5FR catheters of different shapes such as cobra-shaped and hook-shaped (Mikaelsson, Medikrit, Tokyo, Japan), classic J and hydrophilic guide wires 0.035 (Terrumo, Japan) and nonionic Omnipaque contrast agent (Nycomed, Princeton, NJ, USA). For thoracic aorta angiography, a contrast agent was injected by the use of the automated injector (18 $\mathrm{ccm} / 20 \mathrm{ccm} / \mathrm{s}$ ). On selective angiography of aortic branches, contrast medium was manually injected, adjusting the amount and pressure to fluoroscopy-visualized angiogram.

Aortography of thoracic aorta was initially performed in all patients, followed by selective angiography of bronchial and intercostal arteries, and intercostobronchial tree as indicated. The selective arterial embolization was done in $9(81.8 \%)$ patients. In $2(18.2 \%)$ patients, it could not be performed because the aspect of the aorta (thoracic aorta tortuosity) prevented a stable selective placement of the tip of the catheter into the target artery.

Angiography of the subclavian artery branches (thyrocervical trunk, costocervical trunk and internal thoracic artery) was performed in cases where selective angiography of bronchial and intercostal arteries yielded negative results after the detection of pathologic process and its localization determined by diagnostic imaging methods 
and/or bronhoscopy. Due attention was paid to the possible detection of Adamkiewicz artery. Angiographic signs suggesting site of bleeding include hypervascularization, intensive parenchymal opacification, pronounced tortuosity, arterial aneurysms and AV fistulas. The embolization of the feeding artery was performed on the basis of pathologic angiographic characteristics pointing to the localization of bleeding. Upon placing the tip of the catheter safely into the target artery, the embolization was performed under fluoroscopy control by manual injection of mixture of contrast solution $(1 \mathrm{ccm})$ and embolization material, Embosphere (BioSphere Medical Inc., MA, USA), particle size 350-500 $\mu \mathrm{m}$.

The amount of the material injected depended on the patency and filling of the vascular bed of the target artery. The embolization was discontinued when the embolization material ceased to flow out from the catheter, indicating a significant increase in the distal resistance of the embolized artery. Control angiographies were done by manual injection of the contrast medium through the same catheter at a pressure adjusted to the new situation in the embolized artery, under the fluoroscopy control. The catheter was previously rinsed with saline. In addition to control selective angiographies of embolized arteries, angiographies of thoracic arteries were also performed to visualize the possible, previously overlooked pathologically altered arteries that may have presented additional sources of bleeding. In the patients presented, there was no need of pulmonary angiography or use of microcatheters $(\leq 3 \mathrm{~F})$ during the procedure of embolization.

After the procedure, the patient's general condition (blood pressure, ECG, breathing, pain severity) and the site of puncture were monitored for $4 \mathrm{~h}$. Then, the patients in good general condition were transferred to a specialized institution for lung diseas- es, accompanied by the medical personnel, for continuous general condition monitoring and management of underlying disease. The patients were under the regular clinical control for the next 24 months. Depending on the disease dynamics and haemoptysis recurrence patterns, the patients were treated conservatively or were referred for the secondary embolization or some other mode of the active treatment.

\section{Results}

All of the 11 angiograms showed pathologic angiographic findings. Such a finding was present on the angiograms of thoracic aorta in 2/11 (18.2\%) patients with extensive bronchiectasias, and on selective angiograms of the thoracic aorta branches in the remaining 9/11 (81.8\%) patients. The angiographic characteristics of pathologic haemorrhage included hypervascularization and intensive parenchymal opacification in 11/11 (100\%), AV fistulas in 6/11 $(54.5 \%)$, tortuous and hypertrophic arteries in $8 / 11(72.7 \%)$ and aneurysms in $3 / 11$ $(27.3 \%)$ patients. The pathologic process in pulmonary parenchyma was localized on the right and left side in 6/11 (54.5\%) and $5 / 11(45.5 \%)$ patients, respectively.

Embolization proved successful in 9/11 $(81.8 \%)$ patients. In $2 / 11$ (18.2\%) patients, it could not be performed although their thoracic aorta angiograms revealed a pathologic area in the lung parenchyma. A tortuous appearance of thoracic aorta and the pattern of bronchial artery origin prevented a safe manipulation and a stable selective access to the artery showing a pathologic finding. Although BAE was not performed in these two patients, they were included in the analysis because BAE was planned and attempted in these patients as well.

Embolization of intercostobronchial arteries and bronchial artery itself was 


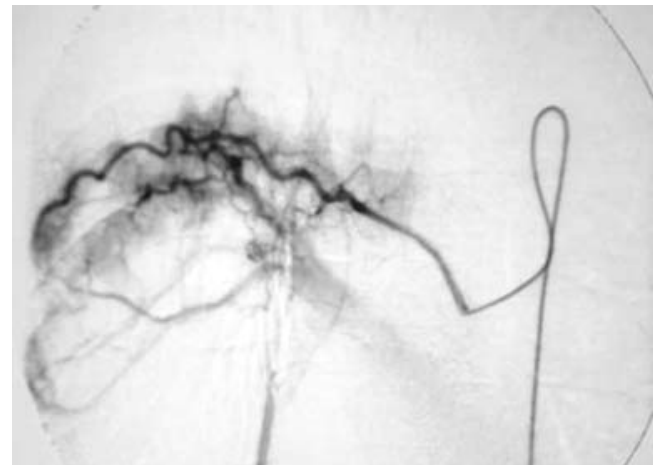

Figure 1a. Digital subtraction angiography in a patient with bronchiectasis before embolization.

done in $8 / 9$ (88.9\%) patients, whereas $1 / 9$ (11.1\%) patient underwent embolization of the thyrocervical trunk branches of the left subclavian artery (ASCL) (Table 1). In five of $8 / 9(62.5 \%)$ patients submitted to selective angiography of bronchial arteries, two bronchial arteries were found on the left side and one bronchial artery on the right side - Type I according to Cauldwell's anatomic classification of the number and origin of bronchial arteries. Adamkiewicz artery as a branch of the arteries involved by the target embolization was not visualized either during or after the procedure; thus, there was no relative contraindication for BAE.

The results of the primary embolization were regularly followed-up for a mean of 21.6 (19-24) months of the procedure (Table 1). The primary procedure proved a successful mode of the treatment for massive haemoptysis in 6/9 (66.7\%) patients. The cause of haemoptysis was lung tuberculosis in $3 / 6(50.0 \%)$ and bronchiectasis in $2 / 6(33.3 \%)$ patients (Figures $1 \mathrm{a}, \mathrm{b})$, whereas one $(16.7 \%)$ patient underwent the primary embolization for microcellular bronchial carcinoma, survived for 5 months of the procedure and died from myocardial infarction. The secondary embolization was performed in 3/9 (33.3\%) patients, at a mean of 3 (1-6) months of the initial proce-

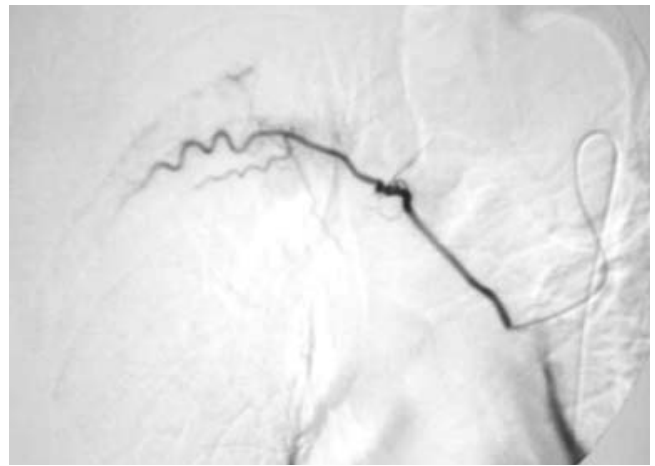

Figure 1b. Digital subtraction angiography in a patient with bronchiectasis after embolization.

dure. In this group, bronchiectasis was the underlying disease in two patients, and aspergilloma in one patient (Figures $2 a, b, c$, $\mathrm{d}, \mathrm{e}$ ). The operative treatment of haemoptysis following two embolization procedures was required in $2 / 9(22.2 \%)$ patients, i.e. for aspergilloma and bronchiectasis in one patient each (Table 1). During the immediate post-embolization procedure (up to 72 h), back pain and coughing were reported by $4 / 9(44.4 \%)$ patients. These complaints were symptomatically treated because any major complications were ruled out by the course of symptoms and respective studies (X-ray, ECG, laboratory tests and CT).

\section{Discussion}

Bronchial arteries are direct parietal branches of the thoracic aorta, originating between $5^{\text {th }}$ and $6^{\text {th }}$ thoracic vertebra at the level of tracheal bifurcation. They supply a nutritive circulation for pulmonary tissue and blood vessels, trachea, inner lung coat, pulmonary hilar lymph nodes, oesophagus and pericardium. Most commonly used classification for describing number and origin of bronchial arteries is from Cauldwell et al. , $^{7}$ According to this classification, two left bronchial arteries and one right presenting as an intercosto- 
Table 1. Results of percutaneous treatment for haemoptysis

\begin{tabular}{|c|c|c|c|c|c|}
\hline Patient No. & Aetiology & $\begin{array}{c}\text { Embolization/period } \\
1 \text { (prim. BAE) }\end{array}$ & $\begin{array}{c}\text { Embolization/period } \\
2 \text { (sec. BAE) }\end{array}$ & Operation & $\begin{array}{c}\text { Control } \\
\text { (months) }\end{array}$ \\
\hline 1 & Tbc & + & & & 20 \\
\hline 2 & Tbc & + & & & 24 \\
\hline 3 & Tbc & - & & & 19 \\
\hline 4 & Asp & + & $+(1$ month $)$ & $+(3$ months $)$ & 22 \\
\hline 5 & Asp & + & & & 19 \\
\hline 6 & Bron & - & & & 23 \\
\hline 7 & Bron & + & $+(2$ months $)$ & $+(4$ months $)$ & 22 \\
\hline 8 & Bron & + & $+(6$ months $)$ & & 23 \\
\hline 9 & Bron & + & & & 21 \\
\hline 10 & Bron & + & & & 23 \\
\hline 11 & $\mathrm{Ca}$ & + & & & $(5-\mathrm{IM})^{\dagger}$ \\
\hline $\mathrm{N}=11$ & & 9/11 (81.9\%) & $3 / 9(33.3 \%)$ & $2 / 9(22.2 \%)$ & 19-24 months \\
\hline
\end{tabular}

Legend: Prim BAE - primary embolization; sec BAE - secondary embolization; Tbc - lung tuberculosis; Asp aspergilloma; Bron - bronchiectasis; Ca - microcellular carcinoma; $\mathrm{N}$ - total patient number; ${ }^{\dagger}$ (IM) myocardial infarction mortality

bronchial trunk (ICBT) (40\%); one on the left and one ICBT on the right (21\%); two on the left and two on the right (one ICBT and one bronchial artery) (20\%); and one on the left and two on the right artery (one ICBT) $(9.7 \%)$. There are also aberrant bronchial arteries (originating outside the level Th5 and Th6) with reported prevalence ranging from $8.3 \%-35 \%{ }^{7,8,9}$ Anomalous origins include aortic arch, mammary artery, subclavian artery, brachiocephalic trunk, thyrocervical trunk, inferior phrenic artery and abdominal aorta. One should be aware of the possible presence of the aberrant bronchial arteries when there is not significant bronchial arterial supply to areas of abnormal pulmonary parenchyma or in the patients with recurrent haemoptysis despite a successful embolization.

The presence of intercostobronchial tree was found in two of our patients with lung tuberculosis $(3 / 11)$ and bronchiectasis $(5 / 11)$ as underlying diseases. In one patient suffering from aspergilloma of the upper lobe of the left lung, branches of the subclavian artery were the predominant source of bleeding.
Angiographic signs suggesting bleeding site include dilated and tortuous bronchial arteries, neovascularity, arterial aneurysms, peribronchial hypervascularity, AV shunting into the pulmonary artery or vein. A contrast extravasation as a specific sign of haemorrhage is rarely seen, with reported prevalence from $3.6-10.7 \%$ because the bleeding from bronchial arteries is slow and intermittent. ${ }^{1,2,10,11}$ The comparison of the signs of bleeding and hypervascularization of the lung parenchyma were indicators of the lesion localization and haemorrhage in all patients $(11 / 11 ; 100 \%)$. Besides those findings, at least one of the following pathognomonic signs was present in all our patients: AV fistulas in 6/11 (54.5\%); tortuous, hypertrophic arteries in 8/11 (72.7\%); and arterial aneurysms in 3/11 (27.3\%) patients. We found hypervascularization of the lung parenchyma and tortuous arteries in 1 (9.1\%) patient with lung carcinoma, which is in contrast to the reports on the absence of angiographic signs of haemoptysis in onethird of individuals with malignant lung diseases. ${ }^{1}$ However, the adequate treatment of haemoptysis in lung cancer patients can 


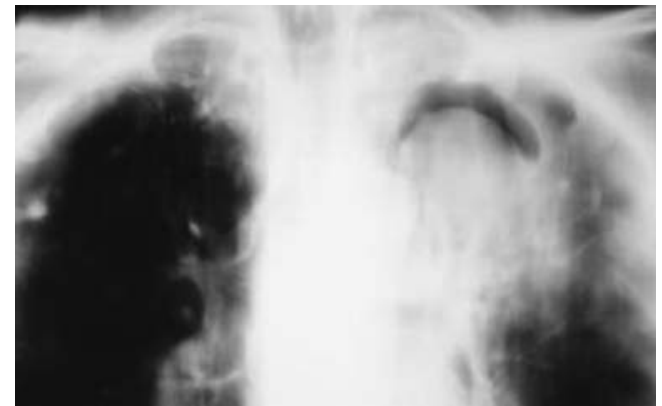

Figure 2a. X-ray/tomography of aspergilloma.

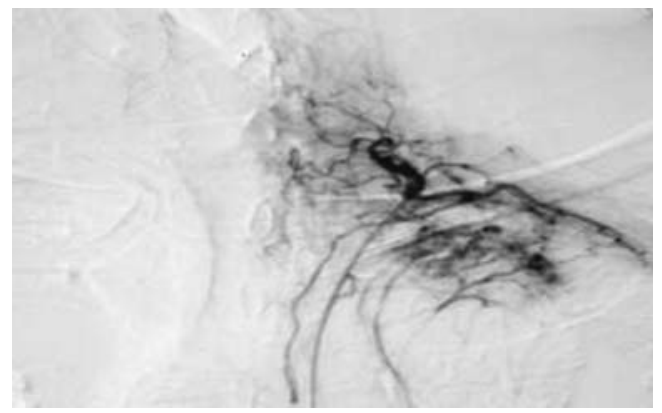

Figure 2c. Digital subtraction angiography in a patient with aspergilloma before embolization.

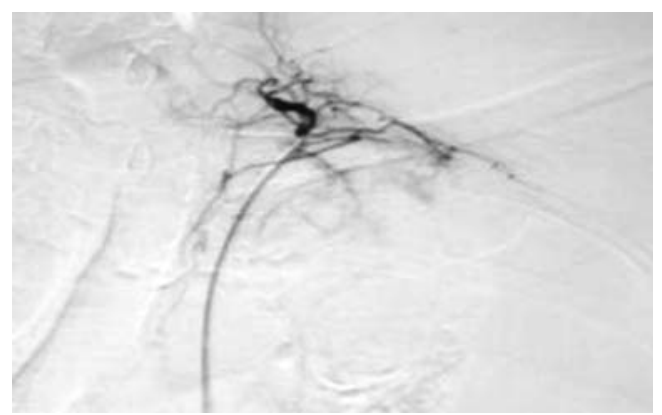

Figure 2e. Digital subtraction angiography in a patient with aspergilloma after secondary embolization.

improve their short-time survival rate, not only because of the patient's characteristics (better performance status, more females), but also because of a more suitable management of urgent conditions. ${ }^{12} \mathrm{AV}$ shunting is a major and reliable angiographic sign of haemoptysis on DSA, recorded in all 5/11 $(45.5 \%)$ patients with bronchiectasis as the underlying disease and in one of the two (9.1\%) patients with aspergilloma.

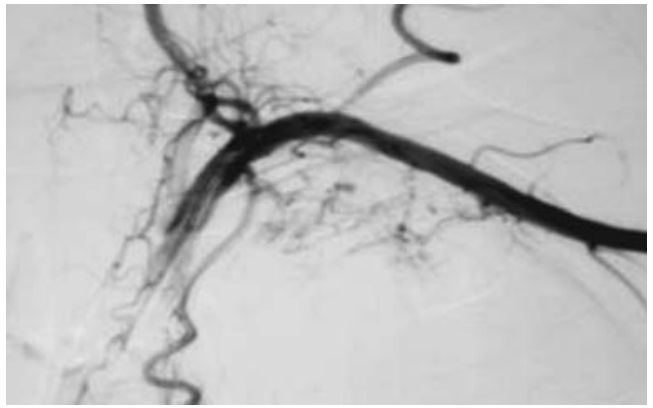

Figure $\mathbf{2 b}$. Digital subtraction angiography in a patient with aspergilloma before embolization.

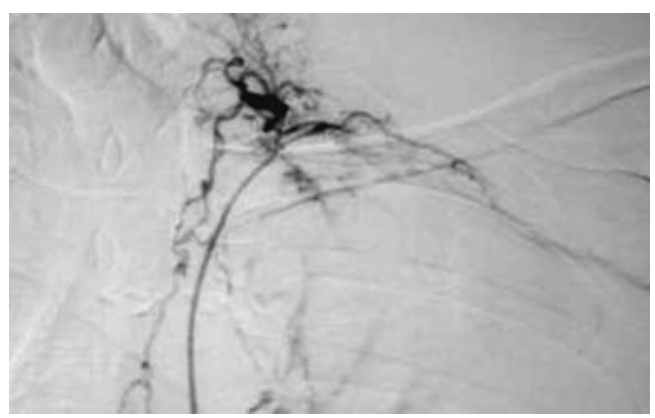

Figure 2d. Digital subtraction angiography in a patient with aspergilloma after primary embolization.

BAE has been used as a therapeutic method of the treatment for haemoptysis since 1973. It is also used as an adjuvant method to the surgical, bronchoscopic and/or conservative treatment. ${ }^{1,2,13,14} \mathrm{BAE}$ enables a direct bleeding control, thus obviating the need of the operative procedure, which is associated with higher mortality $(17.6-19 \%)^{15,16}$, longer hospital stay and higher costs of the treatment. ${ }^{2,4}$ In addition, the embolization provides better clinical preconditions for the possible subsequent operative treatment. In BAE the rate of the technical success is high (77\%-94\%). A longterm success of the primary BAE is up to $80 \%$ and depends on the grade of progression of the underlying pulmonary disease causing hemoptysis. ${ }^{3,14,17}$ In our patient group the success of the percutaneous approach in the management of haemoptysis was $81.9 \%$ (9/11 patients). In nine patients a 
$100 \%$ technical success was recorded. In remaining two patients the embolization was not performed due to the failure of achieving a stable position of the catheter, which is the precondition for performing a safe embolization. The stable selective placement of the tip of the catheter into the target artery could not be achieved because of significant thoracic aorta tortuosity.

In the literature, one-month clinical results of bronchial artery primary embolization range from $48 \%$ to $98 \%{ }^{1,18}$ Such a broad range of the results of the primary embolization may be explained by pathophysiologic changes that occur after the embolization. The embolization is quite frequently followed by hypertrophy of the "adjacent" arteries. The primary procedure failure and proneness to secondary haemorrhages may be caused by the underlying disease activity, circulatory collateralization, or inadequate embolization and prevention of broad communication between the target and adjacent arteries. We presume that the possible inadequate primary embolization may have induced the recurrence in our patient with the apical localization of aspergilloma (Figure 2). Bronchiectasis and lung tuberculosis are the factors that lead to massive haemoptysis and the multiple active treatment (embolization and/or operative procedure) in $73 \%$ of patients. ${ }^{1}$ In our patient series, bronchiectasis and lung tuberculosis contribute around $73 \%$ cases of haemoptysis, the former being found in $45.5 \%$ (5/11) of patients. In the group of patients requiring multiple BAE and/or the operative treatment, bronchiectasis was the underlying disease in two-thirds and aspergilloma in one-third of patients. Lung tuberculosis was not present in this group of patients. This is an interesting finding, considering the fact that lung tuberculosis is the underlying disease in $15.5 \%$ of all haemoptysis recurrences. ${ }^{1}$ The variation between our results and literature data on the prevalence of lung tu- berculosis can be attributed to the small patient sample, making the comparison with other reports imprecise. The small number of patients included in our study prevented any reliable comparison of our data on the high prevalence of bronchiectasis and aspergilloma as the factors for BAE or the operative treatment with the respective literature reports. However, the inappropriate treatment of the underlying disease and/ or disease extension (e.g., aspergilloma in the upper lobe of the left lung; Figure 2) may be objective reasons of the recurrence. This may explain the higher prevalence of multiple embolizations in our patient series (33.3\%) as compared with $18.4 \%$ reported in the literature. The prevalence of lesions detected in non-bronchial arteries in patients with the unsuccessful embolization procedure recorded in our patient series (33.3\%) was comparable to literature reports $(25.0 \%-36.4 \%)$. Chronic inflammatory diseases of pulmonary parenchyma lead to the reduction of pulmonary circulation at the level of pulmonary arterioles due to the associated vasculitis and hypoxic vasoconstriction. This in turn entails the opening of precapillary anastomoses of bronchial and pulmonary arteries, which results in bronchial arterial hypertrophy. The elevation of intravascular pressure or inflammatory erosion of arterial wall in the arteries affected with such lesions usually causes ruptures and consequential haemorrhage. A good control of the underlying disease is the main factor of a long-term success and prevention of haemoptysis recurrence. ${ }^{3,12}$ In our series, chronic inflammatory states (bronchiectasis, aspergilloma) caused the recurrence in three $(33.0 \%)$ patients within 6 months of the primary procedure. The presence of the apical process in the lungs (as in our patient with aspergilloma; Table 1, patient no. 4) make the procedure of the embolization more complex and the therapeutic outcome uncertain. Such a situation requires 
the successful embolization of a number of intrathoracic branches of the subclavian artery and possible collateral arteries as the potential sources of haemorrhage. In the context of bronchiectasis and aspergilloma as the reasons for the secondary treatment in three patients, the BAE procedure was more complex, as demonstrated by the need of a tertiary, operative procedure in two of these patients (one patient with aspergilloma and bronchiectasis each) (Table 1) Prior to the embolization, it is necessary to estimate the distribution pattern of radicular arteries and the origin of anterior spinal artery (artery of Adamkiewicz), involved in the vascularization of spinal medulla, since its embolization leads to spinal ischemia and transverse myelitis.9,19 This unilateral vessel usually arises between Th9 and Th12 in $75 \%$ of cases. Anterior medullary arteries have characteristic "hairpin" configuration at angiography. In our patients, the artery of Adamkiewicz was not visualized on DSA and selective DSA, ensuring a safer approach in the procedure of the embolization. However, during BAE a due attention was paid to the possible visualization of the mentioned arteries because Uflacker et al. report on radicular arteries visualized during the embolization, which may be a contraindication for BAE, in $42 / 75$ cases. ${ }^{20,21}$ However, these authors did not consider it an absolute contraindication for BAE.

In cases of a difficult placement of the tip of a 5FR selective catheter into the target artery, the procedure of BAE can frequently be simplified and safer with the use of microcatheters. Employing a microcatheter is favourable for enabling a more selective access to the target artery and the microcatheter tip placement more distally from the possible radicular artery origin. ${ }^{21}$ However, a safe placement of the tip of 5FR selective catheter into the target artery can be checked by vigorous yet properly dosed injection of a contrast medium, as we did in our patients.
If this does not result in the catheter tip "popping up" from the target artery, the embolization can be performed without the use of microcatheter. Of course, a precondition is ruling out the origin of radicular arteries. That is why we believe, like some other authors, that microcatheters are not necessary to use on BAE in such cases. ${ }^{22}$

The advances in DSA technique, along with the use of microcatheters and nonionic iso-/low-osmolarity contrast agents, have enabled a more efficient and safer approach in the management of haemoptysis. The rate of haemoptysis recurrence may reach $46 \%$ after the initial embolization. According to some authors, in a high percentage $(87 \%)$ it occurs consequentially to recanalization of the previously embolized arteries rather than due to arterial hypertrophy $(27 \%){ }^{21,23}$ This implies to the procedures performed with the use of temporary embolization agent, Gelfoam. ${ }^{20}$ Polyvinyl alcohol (PVA) and Microsphere (Embosphere, Embogold) are permanent embolization agents that require the re-embolization in only $15.5 \%$ of cases. ${ }^{24}$ The procedure success also depends on the choice and type of embolization material needed for the arterial occlusion. 1,2,8,9,18,19 Therefore, we used Embosphere (BioSphere Medical Inc., MA, USA). None of our patients was initially scheduled for the operative procedure. If not so, there would have been no reason to avoid using a temporary embolization agent, followed by the operation. We also avoided using liquid embolic agents (Absolute ethanol, glues) because they cause the occlusion of the capillary network, leading to tissue necrosis. ${ }^{23-25}$ The choice of embolization material is among the most important steps in the procedure of BAE. This primarily refers to the material properties that determine the duration of the embolization effect, and to the size of the particles employed $(350-500 \mu \mathrm{m})$, which taken together ensure a long-term haemoptysis control. ${ }^{1,2,7,18}$ The size of the emboliza- 
tion material particles should be in size of $350-500 \mu \mathrm{m}$. It is essential to avoid use of embolic less than $350 \mu \mathrm{m}$ because it increases the risk of unintentional systemic or pulmonary embolization. Experimental studies have demonstrated a bronchopulmonary anastomosis of $325 \mu \mathrm{m}$ in human lung. ${ }^{26}$

This is of utmost importance because of the width and patency of communications between bronchial arteries and pulmonary veins, and bronchial arteries and pulmonary arteries, as well as for some unexpected communications of bronchial arteries with systemic arteries, also described in other endovascular procedures..$^{18,23,24,27}$ With the use of 350-500 $\mu \mathrm{m}$ Embospheres, we achieved technically satisfactory results in $81.8 \%$ of patients, comparable to literature reports. The rate of the successful outcome of the primary embolization (66.7\%) was also quite consistent with literature data. ${ }^{1-3,7,13,14,17,18,20,21,28}$

In addition to the proper choice of embolization material, the choice of contrast medium is also highly relevant in the procedure of BAE. Hyperosmolarity may cause transient ischemia of radicular arterial branches. Literature reports describe transverse myelitis consequential to vasotoxicity caused by the use of ionic contrast.., 19 Therefore, we employed Omnipaque, a nonionic, low-osmolarity contrast medium, associated with the absence of neurotoxicity and reduced coughing. In spite of this, 4/9 (44.4\%) patients developed cough and back pain within $72 \mathrm{~h}$ of the procedure. Following respective studies, these complaints were treated symptomatically and did not require additional therapy nor prolonged the expected duration of the hospital stay. This is consistent with literature reports on the rate of post-BAE pain development $(24 \%-91 \%){ }^{10}$

\section{Conclusions}

Massive haemoptysis is a clinical emergency, posing a life threat to the patient due to the potential asphyxia. The embolization of bronchial and non-bronchial arteries is a safe and efficient interventional procedure for the successful management of acute haemoptysis. Proper knowledge of the bronchial artery anatomy, their possible communications and underlying pathophysiology of massive haemoptysis are preconditions for the successful procedure performance. Advances in the angiography technique and the use of novel, sophisticated materials make the procedure of BAE safe and associated with a minimal risk for the patient. Due control of the underlying disease, that has led to parenchymal and arterial lesions, thus causing the bleeding, is an important factor to prevent the recurrent bleeding. We do hope that the general notes on the issue of haemoptysis, technique, advantages and results of BAE, and on the role of proper choice of embolization material will result in a more favourable approach to the problem of massive haemoptysis. This is expected to bring an even closer interdisciplinary collaboration of pulmonologists and interventional radiologists, to the benefit of our patients.

\section{References}

1. Yu-Tang Goh P, Lin M, Teo N, En Shen Wong D. Embolization for hemoptysis: a six-year review. Cardiovasc Intervent Radiol 2002; 25: 17-25.

2. Yoon W. Embolic agents used for bronchial artery embolization in massive haemoptysis. Expert Opin Pharmac 2004; 5: 361-7.

3. Kim KJ, Yoo JH, Sung NC, Won HS, Yoou KH, Kang $\mathrm{HM}$. The factors related to recurrence after transcatheter arterial embolization for the treatment of hemoptysis. Korean J Intern Med 1997; 12: 45-51. 
4. Fernando HC, Stein M, Benfield JR, Link DP. Role of bronchial artery embolization in the management of hemoptysis. Arch Surg 1998; 133: 862-6.

5. Hayakawa K, Tanaka F, Tourizuka T, Mitsumori M, Okuno Y, Matsui A, et al. Bronchial artery embolization for hemoptysis: immediate and long-term results. Cardiovasc Intervent Radiol 1992; 15: 154-8.

6. Crocco JA, Rooney JJ, Fankushen DS, DiBenedetto RJ, Lyons HA. Massive hemoptysis. Arch Intern Med 1968; 121: 495-8.

7. Cauldwell EW, Siekert RG, Lininger RE. The bronchial arteries: an anatomic study of 150 human cadavers. Surg Gynecol Obstet 1948; 86: 395-41.

8. Sancho C, Escalante E, Dominguez J, Vidal J, Lopez E, Valldeperas J, Montana XJ. Embolization of bronchial arteries of anomalous origin. Cardiovasc Intervent Radiol 1998; 21: 300-4.

9. Kardijev V, Symeonov A, Chankov I. Etiology, pathogenesis and prevention of spinal cord lesions in selective angiography of bronchial and intercostal arteries. Radiology 1974; 112: 81-3.

10. Ramakantan R, Bandekar VG, Gandhi MS, Aulakh BG, Deshmukh HL. Massive hemoptysis due to pulmonarytuberculosis: control with bronchial artery embolization. Radiology 1996; 200: 691-4.

11. Hsiao EI, Kirsch CM, Kagawa FT, Wehner JH, Jensen WA, Baxter RB. Utility of fiberoptic bronchoscopy before bronchial artery embolization for massive hemoptysis. Am J Roentgenol 2001; 177: 861-7.

12. Debevec L, Jerič T, Kovač V, Bitenc M, Sok M. Is there any progress in routine management of lung cancer patients? A comparative analysis of an institution in 1996 and 2006. Radiol Oncol 2009; 43: 47-53.

13. Remy J, Arnaud A, Fardou H, Giraud R, Volsin C. Treatment of hemoptysis by embolization of bronchial arteries. Radiology 1977; 122: 33-7.

14. Kato A, Kudo S, Matsumoto K, Fukahori T, Shimizu T, Uchino A, et al. Bronchial artery embolization for hemoptysis due to benign disease: immediate and long term results. Cardiovasc Intervent Radiol 2000; 23: 351-7.

15. Conlan AA, Hurwitz SS, Krige L, Nicolaou N, Pool R. Massive hemoptysis. Review of 123 cases. $J$ Thorac Cardiovasc Surg 1983; 85: 120-4.

16. McCollun WB, Mattox KL, Guinn GA, Beall AC Jr. Immediate operative treatment for massive hemoptysis. Chest 1975; 67: 152-5.
17. Mal H, Rullon I, Mellot F, Brugiere O, Sleiman C, Menu $Y$, et al. Immediate and long term results of bronchial artery embolization for life threatening hemoptysis. Chest 1999; 115: 996-1001.

18. Yoon W, Kim JK, Kim YH, Chung TW, Kang HK. Bronchial and nonbronchial systemic artery embolization for life threatening hemoptysis; a comprehensive review. Radiographics 2002; 22: 1395-409.

19. Feigelson HH, Ravin HA. Transverse myelitis following selective bronchial arteriography. Radiology 1965; 85: 663-5.

20. Uflacker R, Kaemmerer A, Picon PD, Rizzon CFC, Neves CMC, Oliveira ESB, et al. Bronchial artery embolization in the management of haemoptysis: technical aspects and long-term results. Radiology 1985; 157: 637-44.

21. Tanaka N, Yamakado K, Murashima S, Takeda K, Matsumara K, Nakagawa T, et al. Superselective bronchial artery embolization for haemoptysis with a coaxial microcatheter system. J Vasc Intervent Radiol 1997; 8: 65-70.

22. Lopez JK, Lee H-Y. Bronchial artery embolization for treatment of life threatening hemoptysis. Semin Intervent Radiol 2006; 23: 223-9.

23. Mauro MA, Jaques PF. Transcatheter bronchial artery embolization for inflammation (hemoptysis). Baum S, Pentecost M, eds. Abrams Angiography. Boston: Lippincott Williams and Wilkins 1997; pp. 819-28.

24. Corr PD. Bronchial artery embolization for life threatening hemoptysis using tris-acryl microspheres; short term results. Cardiovasc Intervent Radiol 2005; 28: 439-41.

25. Marshall TJ, Jackson JE. Vascular intervention in the thorax; bronchial artery embolization for hemoptysis. Eur Radiol 1997; 7: 1221-7.

26. Pump K. Distribution of bronchial arteries in human lung. Chest 1972; 62: 447-51.

27. Rivera-Sanfeliz G, Kansal N. Right thyrocervical trunk bronchial artery collateral: source of type II endoleak after endovascular repair of thoracic aortic aneurysm. J Vasc Intervent Radiol 2007; 18: 655-8.

28. White RI Jr. Bronchial artery embolotherapy for control of acute hemoptysis: analysis of outcome. Chest 1999; 115: 912-5. 